\title{
Clinical studies monitoring circulating and disseminated tumor cells in gastrointestinal cancers
}

\author{
Petra Eliasova $^{1,2}$, Katarina Kolostova ${ }^{1}$, Christopher Kobierzycki ${ }^{3}$, Vladimir Bobek ${ }^{1,2}$ \\ ${ }^{1}$ Department of Tumor Biology, Third Faculty of Medicine, Charles University, Prague, \\ Czech Republic \\ ${ }^{2}$ Department of Surgery, Third Faculty of Medicine, Charles University and University Hospital, \\ Kralovske Vinohrady, Prague, Czech Republic \\ ${ }^{3}$ Department of Histology and Embryology, Wroclaw Medical University, Wroclaw, Poland
}

\begin{abstract}
Circulating tumor cells (CTCs) and disseminated tumor cells (DTCs) are responsible for the development of metastatic disease, and may also hold the key to determining tailored therapies of advanced cancer disease. Our review summarizes the prognostic significance of the detection of CTCs and DTCs in various gastrointestinal cancers with an overview of their possible use as prognostic biomarkers. This could be used in the future as a starting point for new clinical trials focusing on the predictive potential of circulating and disseminated tumor cells. (Folia Histochemica et Cytobiologica 2013, Vol. 51, No. 4, 265-277)
\end{abstract}

Key words: circulating tumor cells; gastrointestinal cancer; esophageal cancer; colorectal cancer; gastric cancer; plastin3; prognosis

\begin{abstract}
Abbreviations
$\mathrm{AFP}$ - alpha fetoprotein; $\mathrm{BM}$ - bone marrow; CD - cluster of differentiation; CEA - carcinoembryonic antigen; CHT - chemotherapy; $\mathrm{CI}$ - confidence interval; CTC - circulating tumor cell; CRC colorectal carcinoma; CVB - central venous blood; CK - cytokeratin; DAPI - 4,6-diamidino-2-phenylindole; DFS — disease free survival; DTC - disseminated tumor cell; EpCAM - epithelial cell adhesion molecule; FISH - fluorescent in situ hybridization; 5-FU - 5-fluorouracil; HCC - hepatocellular carcinoma; HR — hazard ratio; ISET — isolation by size of epithelial tumor; ITC — isolated tumor cells; MACS — magnetic activated cell sorting; MFS — metastasis free survival; MSP — methylation specific polymerase chain reaction; $\mathrm{MVB}$ - mesenteric venous blood; NA - not available; OS - overall survival; $\mathrm{PB}$ - peripheral blood; PFS - progression-free survival; qPCR
\end{abstract}

Correspondence address: V. Bobek, Department of Tumor Biology Third Faculty of Medicine, Charles University Ruska 87, 10097 Prague, Czech Republic

Tel.: +420 267102 108, fax: + 420267102 650;

e-mail:vbobek@centrum.cz
- quantitative real-time polymerase chain reaction; RFA - radiofrequency ablation; RT — radiotherapy; RT-PCR - reverse transcription polymerase chain reaction; TGF $\beta 1$ - transforming growth factor $\beta 1$; TRC method - transcription reverse-transcription concerted method

\section{Introduction}

Single tumor cells occurring in blood circulation are called circulating tumor cells (CTCs), while the single tumor cells seeding distant organs prior to detection of metastasis are termed DTCs (disseminated tumor cells) [1]. CTCs and DTCs are believed to be responsible for the development of metastatic disease, as shown in the parallel-progression model of metastatic cascade [1,2].

Over the last decade, various methods and systems have been developed to isolate and characterize CTCs and DTCs. The presence of these cells accompanies tumor invasion through the bloodstream and dissemination into other distant sites. Much effort has been necessary to understand the biology of cancer dissemination and to make clinical use of CTCs and DTCs. Our review summarizes the prognostic significance of 
the detection of circulating and disseminated tumor cells in various gastrointestinal cancers with a view of their future use in testing processes in clinical studies.

\section{A cancer cell in circulation: a rare event}

Recently, our understanding of cancer has considerably improved. While the basic definition of cancer remains unchanged, it is now considered a complex disease. CTCs and DTCs may be rare events of primary tumor progression. Many clinical studies have been conducted showing the utility of CTC detection in the peripheral blood as a valuable predictor of the clinical outcome for patients with solid tumors [3-5]. Detection, monitoring, and molecular analysis of these extremely rare cancer cells (estimated as one tumor cell per billion normal blood cells in patients with diagnosed metastatic cancer) could provide new possibilities in cancer treatment [6].

The methodology used for CTCs studies in gastrointestinal cancer has been reviewed in depth by Negin et al. [6]. There is no doubt that the development of new more sensitive detection techniques is crucial, and is aimed at gaining higher counts of CTCs and DTCs to make these methods into powerful tools of prediction. We have tried to produce a useful overview of recent methods of detection, isolation, and characterization of CTCs, such as immunomagnetic separation, flow cytometry, fluorescent in situ hybridization (FISH), and reverse transcription polymerase chain reaction (RT-PCR).

Nowadays, the only predictive marker used in colorectal carcinoma (CRC) is the KRAS gene, tested by gene mutational analysis. It is believed that we are close to discovering other genes for predictive purposes. This can also be achieved using CTCs, but their counts seem currently to be insufficient for proper analysis. CTC counts in analyzed peripheral blood in gastrointestinal cancers (e.g., esophageal and gastric cancer), are low compared with other malignancies such as breast and prostate cancer. The absolute numbers in gastrointestinal cancers (such as metastatic colorectal cancer) are reported as $1-2 \mathrm{CTCs} / 7.5 \mathrm{~mL}$ of blood, while in metastatic prostate and breast cancer, counts are on the level of 3-5 and 6-7 CTCs/7.5 mL of blood, respectively [7-10]. It has been discussed that that liver could filter the blood coming in from the peritoneum, so CTCs may remain in the liver and occupy hepatic tissue, developing local metastasis [6]. This could be the reason that significantly higher rates of CTCs can be found analyzing mesenteric venous blood (MVB) in comparison to the peripheral blood [11]. This fact should be reflected in clinical studies, where perioperative blood sampling might be a source of CTCs for predictive analysis.
The range of possible diagnostic and therapeutic uses of CTCs is very wide. Firstly, monitoring cancer disease and demonstrating the therapeutic success achieved by molecular testing of CTCs (which in future may be known as 'liquid biopsy') are possible applications. Secondly, useful methods for inoperable patients where there is no other possibility of obtaining information about the tumor character (which could be called 'real-time tumor biopsy') is another option. In addition, it seems that CTCs and DTCs could provide a very good source of information about the chemosensitivity and chemoresistance of the primary tumor and about distant sites of metastasis [12].

However, very little is still known about the exact number of tumor cells released into the bloodstream by tumors in humans. It is hypothesized that $1 \mathrm{~g}$ of primary tumor may release $10^{6}$ cells into the bloodstream every 24 hours [13]. It has been shown in orthotopic metastatic tumor animal models that surgical manipulation during oncological procedures may enhance the release of cancer cells from the primary tumor site into the circulation. Pressure, biopsy, and laser treatments can all dramatically increase CTC counts (up to sixty-fold), whereas proper tumor resection significantly decreases CTC count [14]. Similarly, increases in CTC counts have been show in human clinical studies of radiofrequency ablation (RFA) - a method of tissue destruction that uses the heat generated by high-frequency alternating current. CTCs from patients with CRC liver metastases were quantified prior to and immediately after open surgery, laparoscopic resection, and open or percutaneous RFA. Surgical procedures led to a statistically significant decrease in CTC counts measured at multiple sites (peripheral vein and artery, hepatic portal vein, hepatic vein). Conversely, RFA, whether open or percutaneous, was associated with a significant increase in CTC count [15]. It may be expected that in vivo detection of intervention-amplified CTCs could be used in the future for early diagnosis of small tumors undetectable with conventional methods [14].

\section{Clinical impact of CTCs in clinical studies in patients with esophageal, gastric, and colorectal cancer}

The clinical relevance of CTC analysis in gastrointestinal cancers is summarized in Tables $1-4$, in which studies are listed according to the diagnosis and the CTC detection method. Table 1 presents immunocytological analysis, whereas Table 2 shows RT-PCR analysis of CTCs in esophageal, gastric, and pancreatic cancers. Similarly, in Tables 3 and 4, immunocytological and RT-PCR based studies of CTCs in colorectal carcinoma are presented. Some of the more interesting results are discussed below. 
Table 1. CTCs/DTCs in esophageal and gastric cancer - immunocytological studies

\begin{tabular}{|c|c|c|c|c|c|c|c|c|c|c|}
\hline $\begin{array}{l}\text { Clinical } \\
\text { Study }\end{array}$ & Year & $\begin{array}{l}\text { Pa- } \\
\text { tients } \\
(n) \\
\end{array}$ & Stage & Sampling & CTC & DTC & $\begin{array}{l}\text { Diagnostic } \\
\text { method }\end{array}$ & OS (months) & $\begin{array}{l}\text { P-value } \\
\text { (OS) }\end{array}$ & Note \\
\hline $\begin{array}{l}\text { Vashist } \\
\text { et al. [18] }\end{array}$ & 2012 & 362 & $\begin{array}{l}\text { Mets, } \\
\text { non } \\
\text {-mets }\end{array}$ & $\begin{array}{l}\text { Pre- and } \\
\text { post- } \\
\text { operative }\end{array}$ & - & Yes & $\begin{array}{l}\text { ICC, } \\
\text { CK assay }\end{array}$ & $\begin{array}{l}\text { DTCs- OS } 39,9 \\
\text { DTCs- DFS } \\
28,2 \\
\text { DTCs+ OS } 13,6 \\
\text { DTCs+ DFS } \\
9,7\end{array}$ & $\begin{array}{l}\mathrm{P}< \\
0.001\end{array}$ & $\begin{array}{l}\text { Esophageal cancer, } \\
\text { DTCs in bone marrow } \\
\text { identified by CK } \\
\text { (cytokeratin), } \\
\text { detailed clinico- } \\
\text { pathologic patient } \\
\text { characteristic }\end{array}$ \\
\hline $\begin{array}{l}\text { Matsu- } \\
\text { saka et } \\
\text { al. [28] }\end{array}$ & 2010 & 52 & $\begin{array}{l}\text { Mets, } \\
\text { non } \\
\text {-mets }\end{array}$ & $\begin{array}{l}\text { At baseline, } \\
\text { during } \\
\text { therapy }\end{array}$ & Yes & - & $\begin{array}{l}\text { Cell- } \\
\text { Search }{ }^{\circledR}\end{array}$ & $\begin{array}{l}2 \text { week point } \\
\text { CTCs- OS } 3,5 \\
\text { CTCs- PFS 4,9 } \\
\text { CTCs + OS } 11,7 \\
\text { CTCs+ PFS } 1,4 \\
4 \text { week point } \\
\text { CTCs- OS 4,0 } \\
\text { CTCs- PFS 5,0 } \\
\text { CTCs+ OS } 11,4 \\
\text { CTCs+ PFS } 1,4\end{array}$ & $\begin{array}{l}\mathrm{P} \leq \\
0.001\end{array}$ & $\begin{array}{l}\text { Gastric cancer, } \\
\text { detailed clinico- } \\
\text { pathologic patient } \\
\text { characteristic }\end{array}$ \\
\hline $\begin{array}{l}\text { Hiraiwa } \\
\text { et al. [7] }\end{array}$ & 2008 & 171 & $\begin{array}{l}\text { Mets, } \\
\text { non } \\
\text {-mets }\end{array}$ & $\begin{array}{l}\text { Pre- and } \\
\text { post- } \\
\text { operative }\end{array}$ & Yes & - & $\begin{array}{l}\text { Cell- } \\
\text { Search }{ }^{\circledR}\end{array}$ & NA & $\begin{array}{l}\mathrm{P}= \\
0.343 \\
(\mathrm{EC}) \\
\mathrm{P}= \\
0.032 \\
(\mathrm{GC})\end{array}$ & $\begin{array}{l}\text { Esophageal cancer } \\
\text { (EC), gastric cancer } \\
\text { (GC) }\end{array}$ \\
\hline $\begin{array}{l}\text { Kolo- } \\
\text { dziejczyk } \\
\text { et al. [44] }\end{array}$ & 2007 & 32 & $\begin{array}{l}\text { Mets, } \\
\text { non } \\
\text {-mets }\end{array}$ & $\begin{array}{l}\text { Before and } \\
\text { after } \\
\text { preoperative } \\
\text { CHT }\end{array}$ & Yes & Yes & IF & $\begin{array}{l}\text { CTCs- 22,6 } \\
\text { CTCs }+20,3\end{array}$ & $\begin{array}{l}P= \\
0.683\end{array}$ & $\begin{array}{l}\text { Gastric cancer } \\
\text { effects of pre- } \\
\text { operative CHT on } \\
\text { CTCs/DTCs }\end{array}$ \\
\hline
\end{tabular}

CHT — chemotherapy; CK — cytokeratin; CTC — circulating tumor cell; DFS — disease-free survival; DTC — disseminated tumor cell; EC — esophageal cancer; GC — gastric cancer; ICC — immunocytochemistry; IF — immunofluorescence; Mets — metastases; NA — not available; OS — overall survival; PFS — progression-free survival

Table 2. CTCs/DTCs in esophageal and gastric cancers - gene expression based studies

\begin{tabular}{|l|l|l|l|l|l|l|l|l|l|l|l|}
\hline $\begin{array}{l}\text { Clinical } \\
\text { Study }\end{array}$ & Year & $\begin{array}{l}\text { Pa- } \\
\text { tients } \\
\text { (n) }\end{array}$ & Stage & Sampling & CTC & DTC & $\begin{array}{l}\text { Dia- } \\
\text { gnostic } \\
\text { method }\end{array}$ & $\begin{array}{l}\text { Mole- } \\
\text { cular } \\
\text { markers }\end{array}$ & $\begin{array}{l}\text { OS } \\
\text { (mon- } \\
\text { ths) }\end{array}$ & $\begin{array}{l}\text { P-value } \\
\text { (OS) }\end{array}$ & Note \\
\hline $\begin{array}{l}\text { Yin et al. } \\
{[19]}\end{array}$ & 2012 & 72 & $\begin{array}{l}\text { Mets, } \\
\text { non } \\
\text {-mets }\end{array}$ & $\begin{array}{l}\text { Pre- and } \\
\text { post- } \\
\text { radiotherapy }\end{array}$ & Yes & - & RT-PCR & $\begin{array}{l}\text { CK19, } \\
\text { CEA, } \\
\text { survivin }\end{array}$ & - & NA & $\begin{array}{l}\text { CTC (+) post-radiothe- } \\
\text { rapy prognostic factor } \\
\text { for ESCC apart from } \\
\text { patients' Karnofsky } \\
\text { performance status } \\
\text { scores. }\end{array}$ \\
\hline $\begin{array}{l}\text { de } \\
\text { Albuqu- } \\
\text { erque et } \\
\text { al. [45] }\end{array}$ & 2012 & 247 & $\begin{array}{l}\text { Non } \\
\text {-mets }\end{array}$ & $\begin{array}{l}\text { Pre- and } \\
\text { post- } \\
\text { operative }\end{array}$ & Yes & - & RT-PCR & $\begin{array}{l}\text { KRT19, } \\
\text { MUC1, } \\
\text { EPCAM, } \\
\text { CE- } \\
\text { ACAM5, } \\
\text { BIRCS, } \\
\text { SCGB2A2, } \\
\text { ERBB2 }\end{array}$ & NA & NA & $\begin{array}{l}\text { CTC (+) } 66.7 \% \text { in } \\
\text { esophageal, 62.2\% in } \\
\text { gastric, } 33.3 \% \text { in small } \\
\text { intestine, } 60.6 \% \text { in co- } \\
\text { lon, and 66.7\% in rectal } \\
\text { adenocarcinomas }\end{array}$ \\
\hline $\begin{array}{l}\text { Hoff- } \\
\text { mann et } \\
\text { al. [46] }\end{array}$ & 2009 & 59 & NA & $\begin{array}{l}\text { Pre- and } \\
\text { post- } \\
\text { operative }\end{array}$ & Yes & - & $\begin{array}{l}\text { Density } \\
\text { gradient, } \\
\text { RT-PCR }\end{array}$ & $\begin{array}{l}\text { Methy- } \\
\text { lated } \\
\text { DAPK } \\
\text { or APC } \\
\text { promoter }\end{array}$ & poor & P = 0.04 & - \\
\hline
\end{tabular}


Table 2. $\mathrm{cd}$.

\begin{tabular}{|c|c|c|c|c|c|c|c|c|c|c|c|}
\hline $\begin{array}{l}\text { Braben- } \\
\text { der et al. } \\
{[47]}\end{array}$ & 2008 & 29 & $\begin{array}{l}\text { Non } \\
\text {-mets }\end{array}$ & $\begin{array}{l}\text { Prior } \\
\text { neoadjuvant } \\
\text { CHT }\end{array}$ & Yes & - & $\begin{array}{l}\text { Density } \\
\text { gradient, } \\
\text { RT-PCR }\end{array}$ & ERCC1 & NA & NA & $\begin{array}{l}\text { ERCC1 mRNA } \\
\text { expression } \\
\text { associated with } \\
\text { response to } \\
\text { neoadjuvant RT }\end{array}$ \\
\hline $\begin{array}{l}\text { Hoff- } \\
\text { mann } \\
\text { et al. [48] }\end{array}$ & 2007 & 62 & NA & $\begin{array}{l}\text { Pre- and post- } \\
\text { operative }\end{array}$ & Yes & - & $\begin{array}{l}\text { Density } \\
\text { gradient, } \\
\text { RT-PCR }\end{array}$ & Survivn & NA & $\mathrm{P}<0.04$ & $\begin{array}{l}\text { Survivin mRNA } \\
\text { levels fall after } \\
\text { surgical resection }\end{array}$ \\
\hline $\begin{array}{l}\text { Liu et al. } \\
{[17]}\end{array}$ & 2007 & 53 & $\begin{array}{l}\text { Non } \\
\text {-mets }\end{array}$ & $\begin{array}{l}\text { Pre- and post- } \\
\text { operative, } \\
\text { 3rd post- } \\
\text { operative day }\end{array}$ & Yes & - & $\begin{array}{l}\text { Density } \\
\text { gradient, } \\
\text { RT-PCR }\end{array}$ & CEA & NA & $\mathrm{P}<0.05$ & $\begin{array}{l}\text { Patients with high } \\
\text { levels CEA in CTC } \\
\text { fraction showed } \\
\text { mets } 1 \text { year after } \\
\text { surgery more often }\end{array}$ \\
\hline $\begin{array}{l}\text { Ikoma } \\
\text { et al. [49] }\end{array}$ & 2007 & 44 & $\begin{array}{l}\text { Mets, } \\
\text { non } \\
\text {-mets }\end{array}$ & Preoperative & Yes & - & $\begin{array}{l}\text { RT-PCR, } \\
\text { MSP }\end{array}$ & $\begin{array}{l}\text { p16, E- } \\
\text { cadherin, } \\
\text { RARbeta }\end{array}$ & NA & $P=0.05$ & $\begin{array}{l}\text { Methylation- } \\
\text {-specific PCR } \\
\text { (MSP) }\end{array}$ \\
\hline $\begin{array}{l}\text { Hoff- } \\
\text { mann } \\
\text { et al. [50] }\end{array}$ & 2007 & 44 & NA & Postoperative & Yes & - & $\begin{array}{l}\text { Density } \\
\text { gradient } \\
\text { (On- } \\
\text { coQuick) } \\
\text { RT-PCR }\end{array}$ & Survivin & NA & $\mathrm{P}<0.04$ & $\begin{array}{l}\text { Gastric, esophageal } \\
\text { CRC, pancreatic: } \\
\text { survivin levels fall } \\
\text { after complete } \\
\text { surgical resection }\end{array}$ \\
\hline $\begin{array}{l}\text { Ikeguchi } \\
\text { et al. [51] }\end{array}$ & 2005 & 59 & NA & $\begin{array}{l}\text { Pre- and post- } \\
\text { operative }\end{array}$ & Yes & - & RT-PCR & CEA & NA & $P=0.064$ & Gastric cancer \\
\hline $\begin{array}{l}\text { Ito et al. } \\
{[52]}\end{array}$ & 2004 & 28 & NA & NA & Yes & - & RT-PCR & $\begin{array}{l}\text { CEA, } \\
\text { CK20 } \\
\end{array}$ & NA & NA & - \\
\hline $\begin{array}{l}\text { Kaganoi } \\
\text { et al. [53] }\end{array}$ & 2004 & 70 & NA & $\begin{array}{l}\text { Pre-, intra- } \\
\text { and post- } \\
\text { operative }\end{array}$ & Yes & - & RT-PCR & SCCA & NA & $\mathrm{P}<0.001$ & $\begin{array}{l}\text { Squamous cell } \\
\text { carcinoma } \\
\text { Antigen (SCCA) }\end{array}$ \\
\hline $\begin{array}{l}\text { Huang } \\
\text { et al. [54] }\end{array}$ & 2003 & 62 & NA & Preoperative & Yes & - & RT-PCR & $\begin{array}{l}\text { CEA, } \\
\text { CK19, } \\
\text { CK20 }\end{array}$ & NA & NA & $\begin{array}{l}\text { Gastrointestinal } \\
\text { cancer }\end{array}$ \\
\hline $\begin{array}{l}\text { Nakashi- } \\
\text { ma et al. } \\
{[55]}\end{array}$ & 2003 & 54 & NA & Preoperative & Yes & - & RT-PCR & CEA & NA & NA & - \\
\hline $\begin{array}{l}\text { Koike } \\
\text { et al. [56] }\end{array}$ & 2002 & 33 & $\begin{array}{l}\text { Mets, } \\
\text { non } \\
\text {-mets }\end{array}$ & $\begin{array}{l}\text { Pre-, intra- } \\
\text { and post- } \\
\text { operative, } \\
1 \text { week after } \\
\text { surgery }\end{array}$ & Yes & - & RT-PCR & $\begin{array}{l}\text { Del- } \\
\text { taNp63 }\end{array}$ & NA & NA & - \\
\hline $\begin{array}{l}\text { Miyazo- } \\
\text { no et al. } \\
{[57]}\end{array}$ & 2001 & 57 & $\begin{array}{l}\text { Mets, } \\
\text { non } \\
\text {-mets }\end{array}$ & $\begin{array}{l}\text { Pre- and post- } \\
\text { operative }\end{array}$ & Yes & - & RT-PCR & CEA & NA & NA & $\begin{array}{l}\text { Gastric cancer, } \\
\text { surgical manipu- } \\
\text { lation }\end{array}$ \\
\hline $\begin{array}{l}\text { Soeth } \\
\text { et al. [58] }\end{array}$ & 1997 & 245 & $\begin{array}{l}\text { Mets, } \\
\text { non } \\
\text {-mets }\end{array}$ & Preoperative & $\begin{array}{l}\text { Yes, } \\
104\end{array}$ & $\begin{array}{l}\text { Yes, } \\
141\end{array}$ & RT-PCR & $\begin{array}{l}\text { CK20 for } \\
\text { DTCs }\end{array}$ & $\begin{array}{l}\text { CK20 } \\
\text { RNA+ } \\
\text { shorter } \\
\text { OS }\end{array}$ & $\begin{array}{l}\mathrm{P}>0.0001 \\
(\mathrm{CRC}) \\
\mathrm{P}=0.0414 \\
(\mathrm{GC}) \\
\mathrm{NA}(\mathrm{PC})\end{array}$ & $\begin{array}{l}\text { Gastric cancer } \\
\text { (GC), colorectal } \\
\text { cancer (CRC), } \\
\text { pancreatic cancer } \\
\text { (PC) }\end{array}$ \\
\hline
\end{tabular}

CHT, CK, CTC, DFS, DTC, GC, Mets, NA, OS - same as described for Table 1. BIRCs - BIR-containing proteins; CEA — carcinoembryonic antigen; CEACAM5 - carcinoembryonic antigen-related cell adhesion molecule 5; CRC - colorectal cancer; DAPK - death-associated protein kinase; ESCC - esophageal squamous cell carcinoma; EpCAM — epithelial cell adhesion molecule; ERBB2 - erythroblastic leukemia viral oncogene homolog 2; ERCC1 - excision repair cross-complementing 1 protein; MSP - methylation specific polymerase chain reaction; MUC1 mucin 1; PC — pancreatic cancer; RT-PCR - reverse transcription polymerase chain reaction; SCGB2A2 - secretoglobin family $2 \mathrm{~A}$ member 2. 


\begin{tabular}{|c|c|c|c|c|c|c|c|c|}
\hline 芩 & 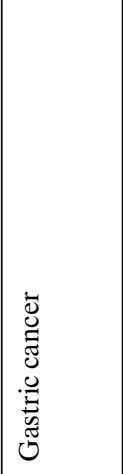 & 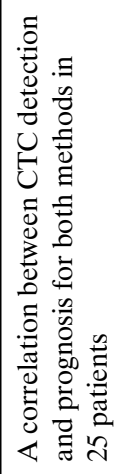 & 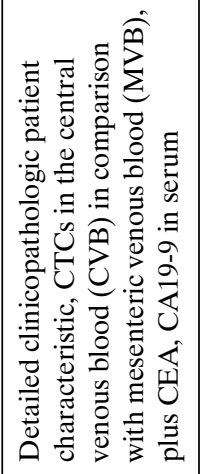 & 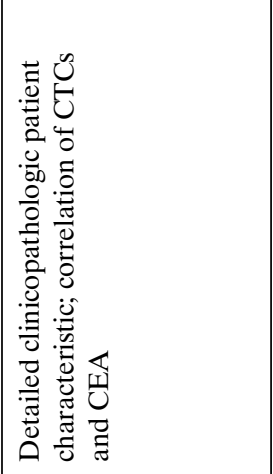 & 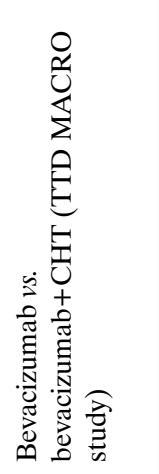 & 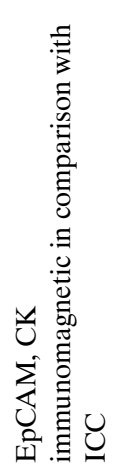 & 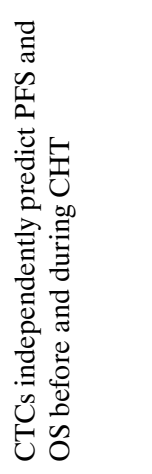 & 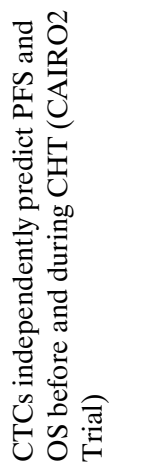 \\
\hline 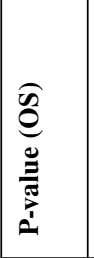 & \begin{tabular}{|l}
0 \\
0 \\
0 \\
0 \\
.$\Xi$ \\
$\dot{3}$ \\
\end{tabular} & 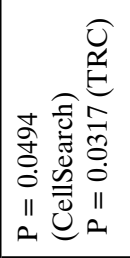 & 艺 & $\begin{array}{l}\tilde{\Xi} \\
0 \\
11 \\
\tilde{0}\end{array}$ & 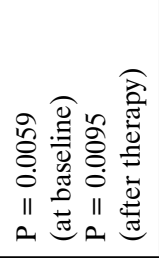 & 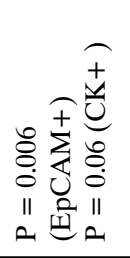 & $\begin{array}{l}\vec{\Xi} \\
\dot{0} \\
v \\
v\end{array}$ & $\begin{array}{l}\tilde{\Xi} \\
\vdots \\
0 \\
v \\
0\end{array}$ \\
\hline 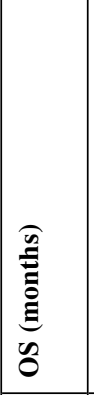 & 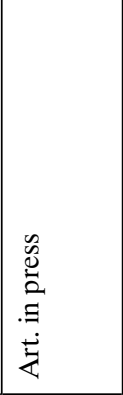 & $\overleftrightarrow{z}$ & 艺 & 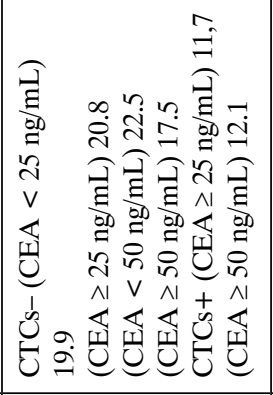 & 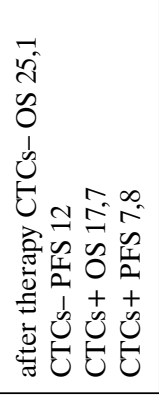 & 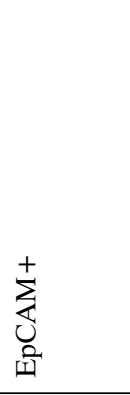 & 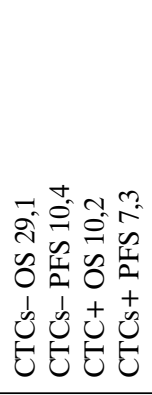 & 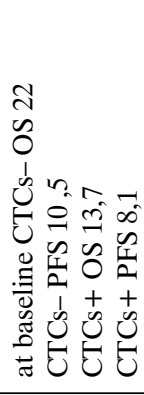 \\
\hline 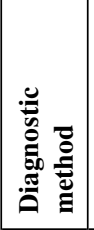 & 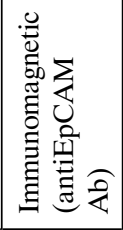 & 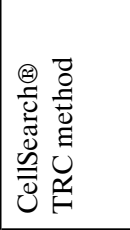 & 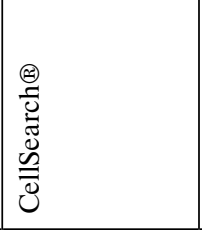 & 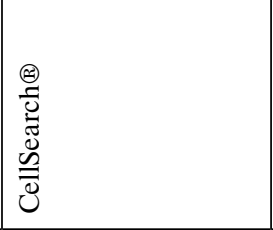 & 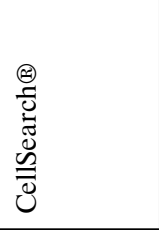 & 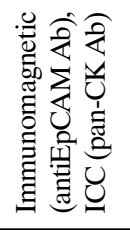 & 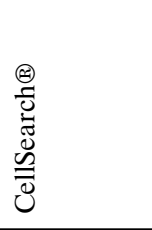 & 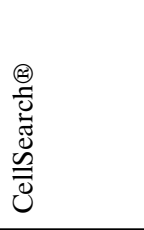 \\
\hline U. & 1 & 1 & 1 & 1 & 1 & 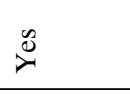 & 1 & 1 \\
\hline U & 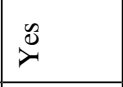 & 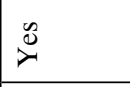 & 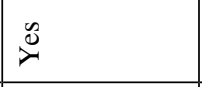 & $\ddot{y}$ & $\stackrel{0}{2}$ & 1 & $\stackrel{\infty}{\nu}$ & $\stackrel{\infty}{\nu}$ \\
\hline 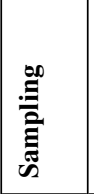 & 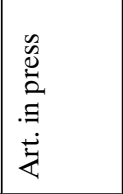 & $\overleftrightarrow{z}$ & 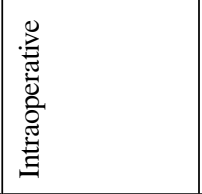 & 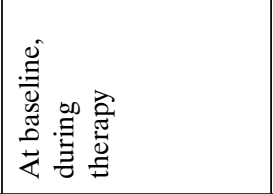 & 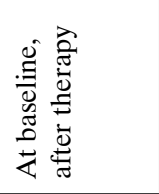 & 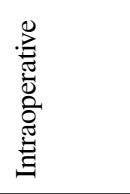 & 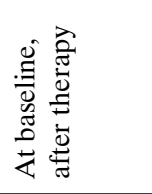 & 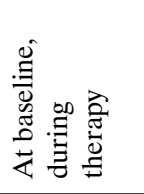 \\
\hline 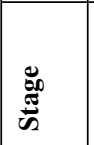 & 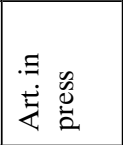 & 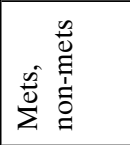 & 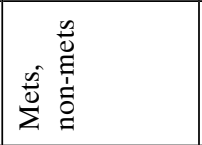 & $\frac{n}{2}$ & $\frac{n}{2}$ & 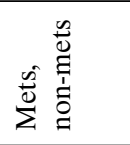 & $\stackrel{n}{e^{\frac{n}{2}}}$ & 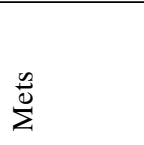 \\
\hline 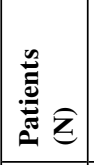 & 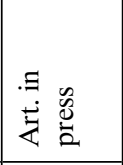 & F & 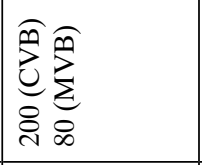 & 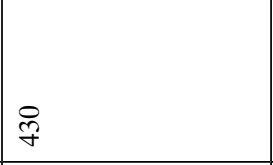 & $\stackrel{\infty}{\sim}$ & $\tilde{\gamma}$ & t & $\vec{y}$ \\
\hline 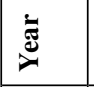 & $\stackrel{m}{\stackrel{\sim}{\sim}}$ & $\stackrel{\sim}{\stackrel{\sim}{*}}$ & ๙ิ) & ָे & $\stackrel{\sim}{\stackrel{\sim}{*}}$ & $\overrightarrow{\widetilde{\sim}}$ & $\overrightarrow{\vec{\sim}}$ & 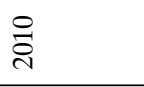 \\
\hline 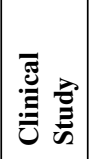 & 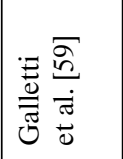 & 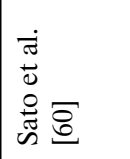 & 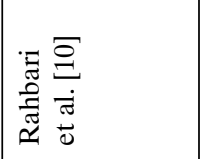 & 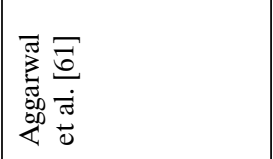 & 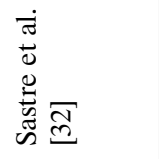 & 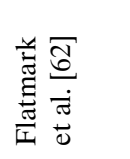 & 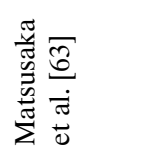 & $\begin{array}{l}\frac{\dot{\sigma}}{\tilde{\sigma}} \\
\stackrel{\overrightarrow{0}}{0} \\
\stackrel{\theta}{\sigma}\end{array}$ \\
\hline
\end{tabular}


Petra Eliasova et al.

\begin{tabular}{|c|c|c|c|c|c|c|c|c|}
\hline 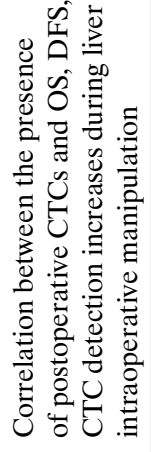 & 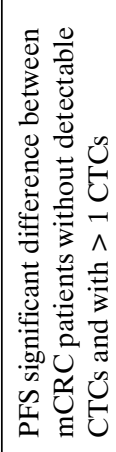 & 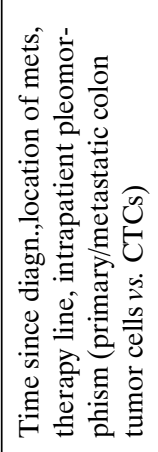 & 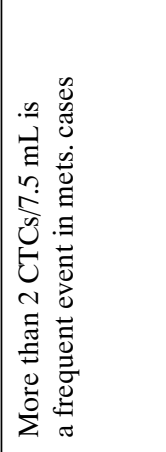 & 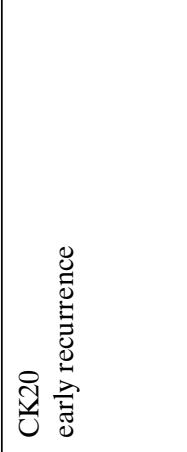 & 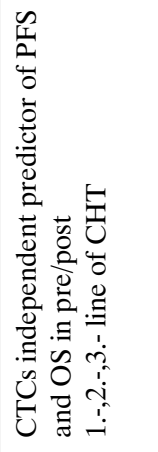 & 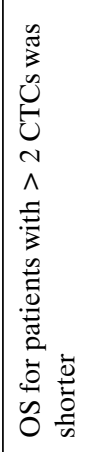 & 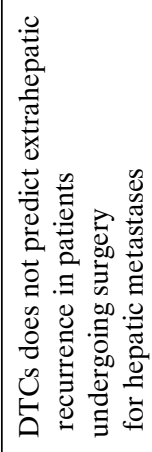 & 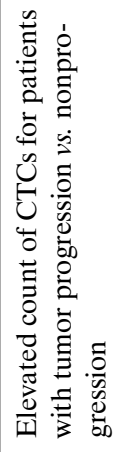 \\
\hline $\begin{array}{l}\text { రొ } \\
0 \\
0 \\
\text { II } \\
0 .\end{array}$ & 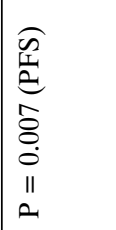 & $\mathbb{z}$ & \begin{tabular}{|l}
$\vec{\Xi}$ \\
$\dot{o}$ \\
$\dot{0}$ \\
$v$ \\
2
\end{tabular} & $\begin{array}{l}\tilde{\Xi} \\
\dot{d} \\
v \\
2 \\
\alpha\end{array}$ & $\begin{array}{l}\vec{\Xi} \\
\dot{o} \\
\dot{0} \\
v \\
\alpha\end{array}$ & $\begin{array}{l}0 \\
0 \\
0 \\
0 \\
11 \\
\alpha\end{array}$ & $\overleftrightarrow{z}$ & $\begin{array}{l}\tilde{\Xi} \\
0 \\
0 \\
\text { II } \\
\alpha\end{array}$ \\
\hline $\mathbb{z}$ & $\overleftrightarrow{z}$ & 艺 & $\mathbb{z}$ & $\overleftrightarrow{\mathrm{z}}$ & 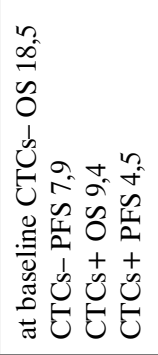 & $\overleftrightarrow{z}$ & $\overleftrightarrow{z}$ & $\overleftrightarrow{z}$ \\
\hline 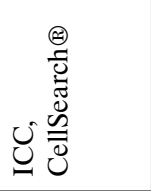 & $\underset{U}{0}$ & 里 & 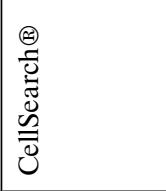 & 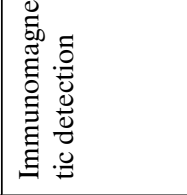 & 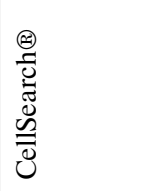 & 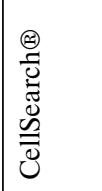 & 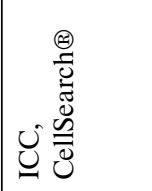 & $\underset{U}{\circlearrowright}$ \\
\hline$y^{y}$ & 1 & 1 & 1 & 1 & 1 & 1 & $\stackrel{\infty}{\infty}$ & 1 \\
\hline$\stackrel{\infty}{\infty}$ & $\stackrel{0}{\nu}$ & $\stackrel{\theta}{\nu}$ & $\stackrel{0}{0}$ & $\stackrel{\theta}{\nu}$ & $\stackrel{\infty}{\nu}$ & $\stackrel{\infty}{\infty}$ & $\stackrel{\theta}{\nu}$ & $\stackrel{y}{\infty}$ \\
\hline 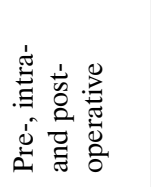 & 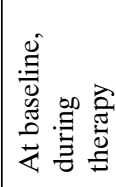 & 总 & 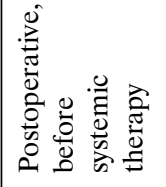 & 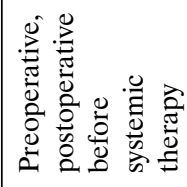 & 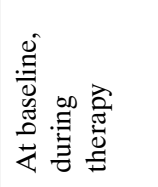 & 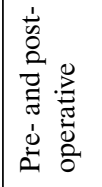 & 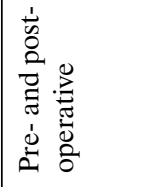 & 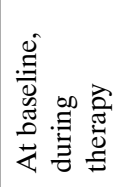 \\
\hline 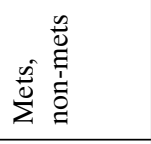 & $\sum^{\frac{n}{2}}$ & $\sum^{\frac{n}{2}}$ & 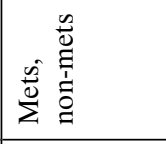 & 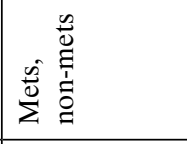 & $\sum_{\Sigma}^{\frac{n}{\omega}}$ & 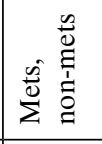 & $\sum^{\frac{n}{\omega}}$ & $\sum_{\Sigma}^{\frac{n}{0}}$ \\
\hline ㄱ & $\infty$ & in & $\stackrel{2}{2}$ & $\stackrel{2}{2}$ & $\underset{\mathscr{r}}{\stackrel{P}{r}}$ & 도 & f & $g$ \\
\hline 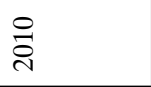 & $\stackrel{\circ}{\stackrel{\sim}{\sim}}$ & 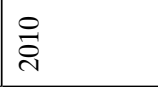 & ڤ્ণ & ڤ્ণ & $\stackrel{\infty}{\stackrel{\sim}{0}}$ & 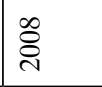 & ๕્ત & ๕્ત \\
\hline 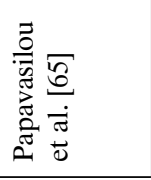 & 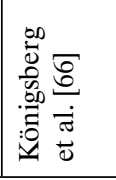 & 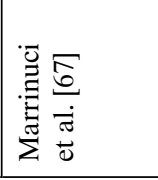 & 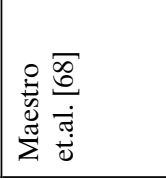 & $\begin{array}{l}\dot{\pi} \\
0 \\
0 \\
00 \\
00 \\
30 \\
3\end{array}$ & $\begin{array}{l}\dot{\pi} \\
\overline{0} \\
0 \\
\overline{0} \\
0 \\
0 \\
0\end{array}$ & 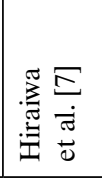 & 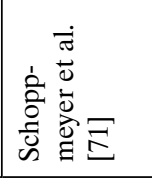 & $\begin{array}{l}\dot{\pi} \\
\overline{0} \\
\overline{0} \\
\overline{0} \\
0 \overline{0}\end{array}$ \\
\hline
\end{tabular}




\begin{tabular}{|c|c|c|c|c|c|c|c|c|c|c|c|}
\hline 愛 & 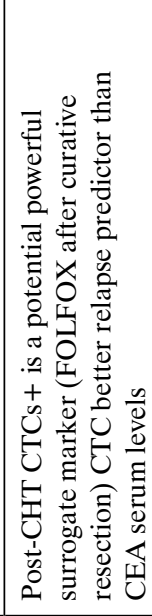 & 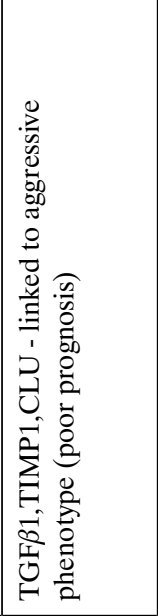 & 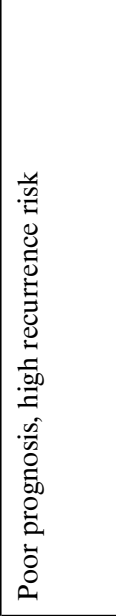 & 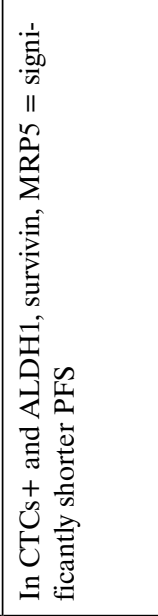 & 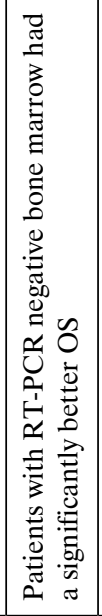 & 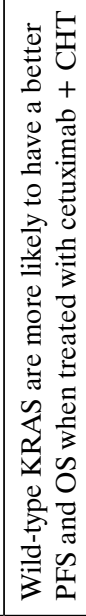 & 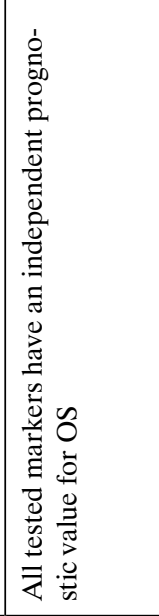 & 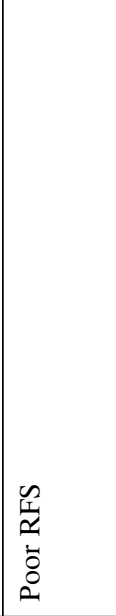 & 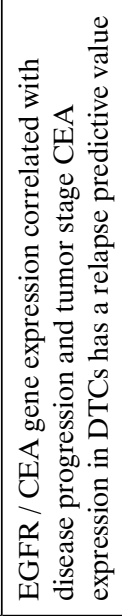 & 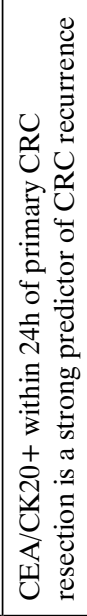 & $\begin{array}{l}n \\
\text { I } \\
\alpha \\
0 \\
0 \\
0 \\
2\end{array}$ \\
\hline 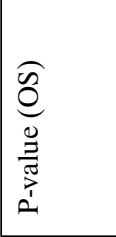 & 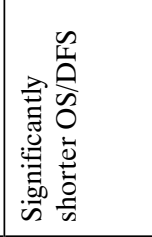 & 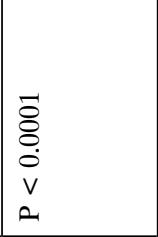 & $\begin{array}{l}\vec{s} \\
\dot{0} \\
v \\
v \\
0\end{array}$ & 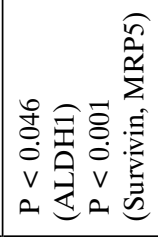 & \begin{tabular}{|l}
$\delta$ \\
$\delta$ \\
0 \\
0 \\
II \\
م.
\end{tabular} & 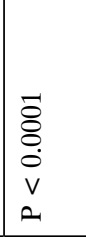 & \begin{tabular}{|l} 
In \\
0 \\
0 \\
0 \\
11 \\
2
\end{tabular} & 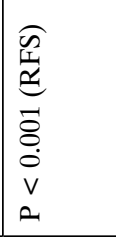 & $\overleftrightarrow{z}$ & $\overleftrightarrow{z}$ & 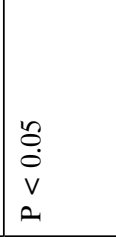 \\
\hline 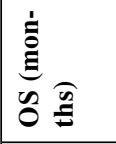 & $\overleftrightarrow{Z}$ & $\overleftrightarrow{z}$ & $\mathbb{z}$ & 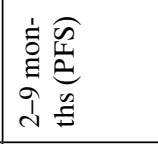 & $\mathbb{z}$ & $\mathbb{z}$ & 艺 & $\mathbb{z}$ & $\overleftrightarrow{z}$ & $\overleftrightarrow{z}$ & 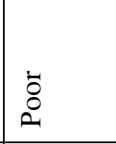 \\
\hline 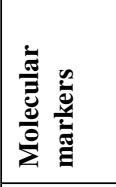 & 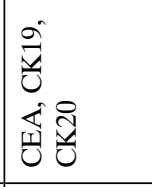 & 焉 & 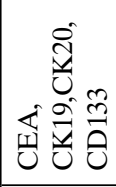 & 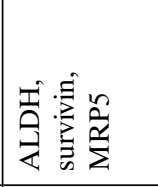 & ปี & 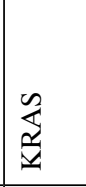 & 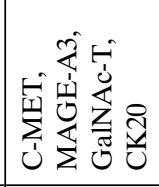 & 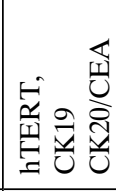 & 式密 & 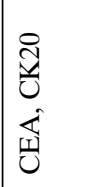 & 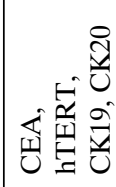 \\
\hline 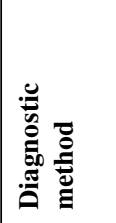 & 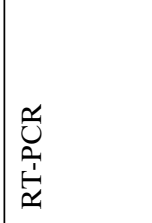 & 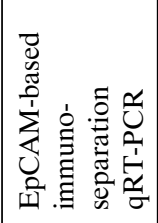 & $\begin{array}{l}0 \\
0 \\
0 \\
\dot{1} \\
\end{array}$ & 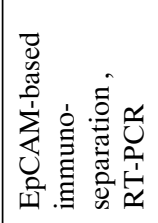 & 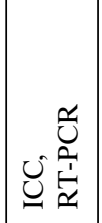 & 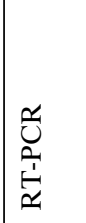 & $\begin{array}{l}\text { Uै } \\
\text { ڤ్ }\end{array}$ & 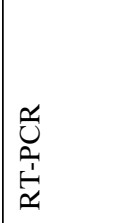 & 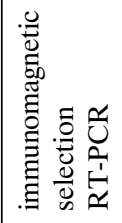 & 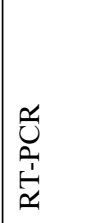 & 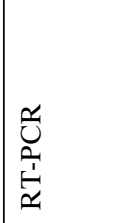 \\
\hline U & 1 & 1 & 1 & 1 & $\stackrel{\Delta}{\circlearrowright}$ & 1 & I & 1 & $\stackrel{\mathscr{s}}{\nu}$ & 1 & 1 \\
\hline U & $\stackrel{\circlearrowright}{\varnothing}$ & $\stackrel{\circlearrowright}{\circlearrowright}$ & $\stackrel{\circlearrowright}{\check{\nu}}$ & 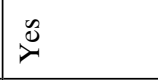 & 1 & $\stackrel{\infty}{2}$ & $\stackrel{\circlearrowright}{\circlearrowright}$ & $\stackrel{\circlearrowright}{\check{\nu}}$ & 1 & 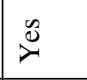 & $\stackrel{\circlearrowright}{2}$ \\
\hline 照 & 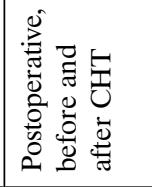 & $\overleftrightarrow{z}$ & 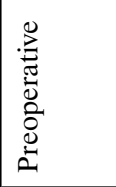 & 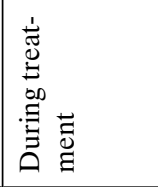 & 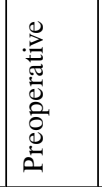 & 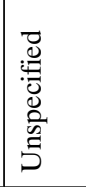 & 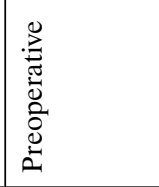 & 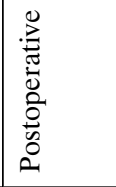 & 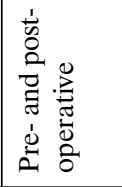 & 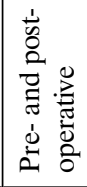 & 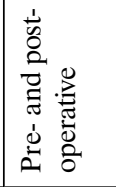 \\
\hline 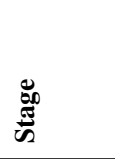 & 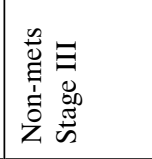 & $\frac{n}{\tilde{e}}$ & 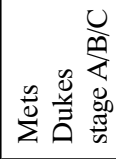 & 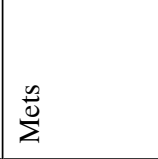 & $\frac{\infty}{e^{0}}$ & $\frac{n}{2}$ & 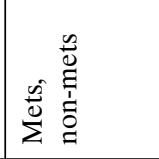 & 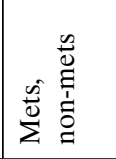 & 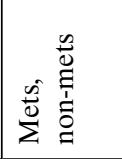 & $\overleftrightarrow{z}$ & 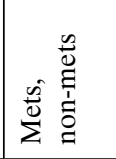 \\
\hline 吾气 & \& & \pm & $\stackrel{\mathscr{n}}{\sim}$ & q & f & $\div$ & मे & $\underset{⿱ 亠 凶}{\mathscr{\vartheta}}$ & $\stackrel{2}{1}$ & $\stackrel{\circ}{\circ}$ & $\hat{n}$ \\
\hline ت્ّ & min & בี & $\overrightarrow{\text { ते }}$ & 올 & 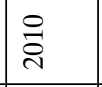 & ڤ్ेે & ڤે̊ & 客 & ڤ્ণ & ڤ్తి & ڤิ) \\
\hline 窇导 & 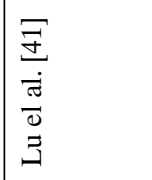 & 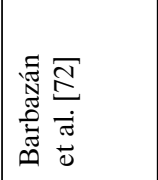 & 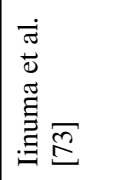 & 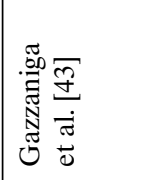 & 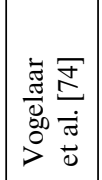 & 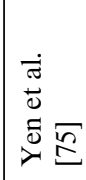 & 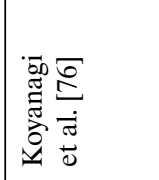 & 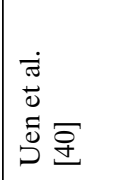 & 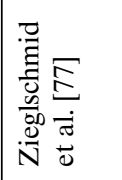 & 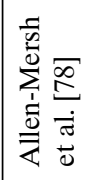 & 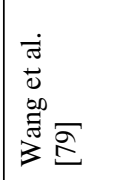 \\
\hline
\end{tabular}




\begin{tabular}{|c|c|c|c|c|c|c|c|}
\hline 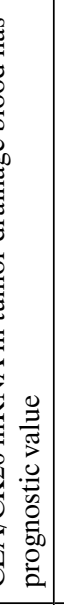 & 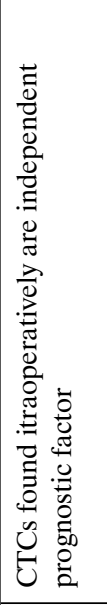 & 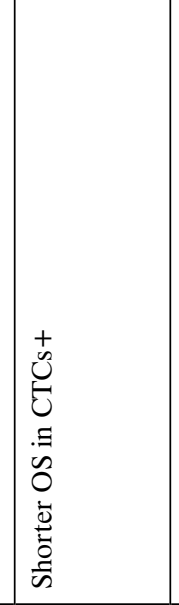 & 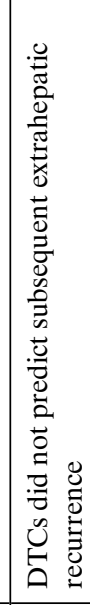 & \begin{tabular}{|c|c} 
\\
\\
\\
\\
0 \\
0 \\
0 \\
0 \\
0 \\
0
\end{tabular} & 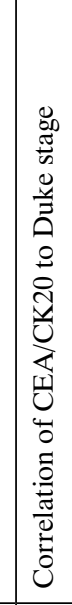 & 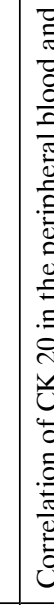 & 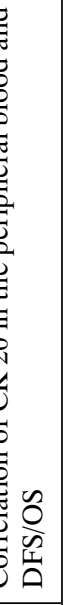 \\
\hline 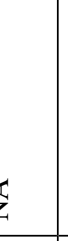 & 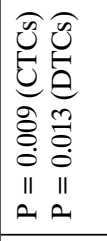 & $\mathbb{z}$ & $\mathbb{z}$ & $\begin{array}{l}8 \\
0 \\
0 \\
11 \\
n\end{array}$ & $\mathbb{z}$ & $\frac{\pi}{z}$ & 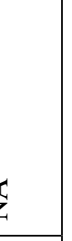 \\
\hline E & $\mathbb{z}$ & 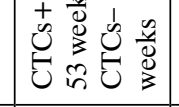 & $\mathbb{z}$ & $\mathbb{z}$ & $\mathbb{z}$ & $\mathbb{z}$ & \\
\hline 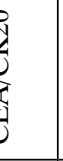 & च̃ & चิ & ฮิ & 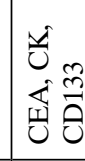 & 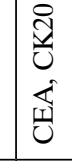 & घี & 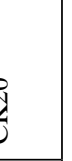 \\
\hline & 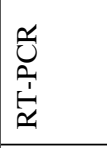 & 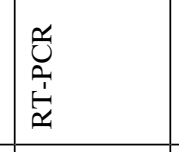 & 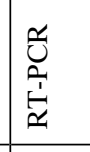 & 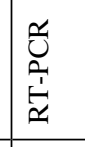 & 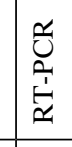 & 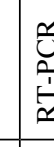 & tיt \\
\hline & $\overbrace{}^{\circ}$ & I & 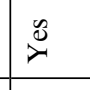 & । & 1 & 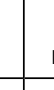 & \\
\hline 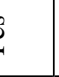 & $\stackrel{\mathscr{c}}{\nu}$ & $\overbrace{\lambda}^{\infty}$ & $y_{x}^{2}$ & $\underset{\gamma}{\mathscr{2}}$ & $\overbrace{\nu}^{\infty}$ & 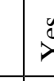 & 8 \\
\hline 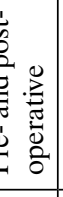 & 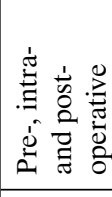 & 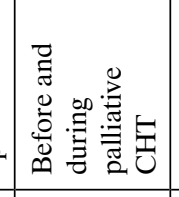 & 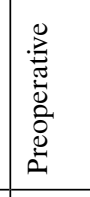 & 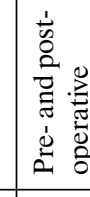 & 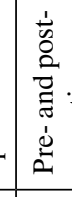 & 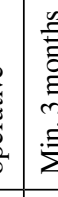 & 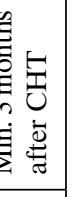 \\
\hline 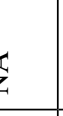 & $\frac{\substack{0 \\
\frac{\omega}{2}}}{2}$ & $\frac{a_{0}^{\frac{0}{2}}}{2}$ & $\frac{\substack{0 \\
\frac{0}{2}}}{2}$ & $\mathbb{z}$ & 密 & $\frac{\frac{5}{0}}{2}$ & 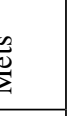 \\
\hline E & 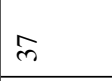 & F & $F$ & 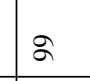 & 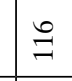 & ¿́ & $\theta$ \\
\hline 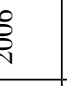 & ڤ్ & पे & 帘 & క్ర & ప్రి & 号 & 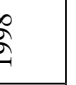 \\
\hline$\underset{\tilde{\sigma}}{\vec{\sigma}}$ & 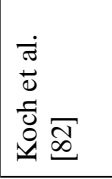 & 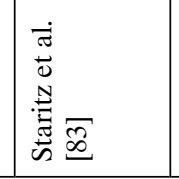 & 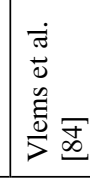 & 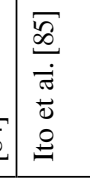 & 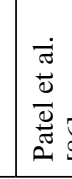 & $\frac{7}{2}$ & 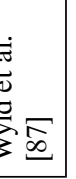 \\
\hline
\end{tabular}




\section{Esophageal cancer (EC)}

Esophageal cancer (EC) is notorious for its aggressive biological behavior, local infiltration, involvement of adjacent lymph nodes, and broad metastasis through hematogenous spread. It has been reported that the frequency of hematogenous recurrence is high, despite radical surgery with lymph node dissection [16]. In this regard, the detection of cancer cells in the blood could be important for identifying patients with a high risk of relapse. There have been many studies showing a positive correlation between detection of CTCs, tumor staging, and patient prognosis. Detection of CTCs from PB of EC patients by conventional qPCR methods has been reported for several genes.

Liu et al. [17] aimed at establishing a quantitative system for evaluating the role of CTCs in PB from patients who underwent surgical resection during esophageal cancer treatment. 155 PB samples from 53 EC patients were collected before surgery (B-1), immediately after surgery (B0), and on the third postoperative day $(\mathrm{B}+3)$. A direct $\mathrm{qPCR}$ method based on CEA mRNA gene expression was designed for the detection of CTCs. The authors showed significant differences between groups B-1 vs. B0 $(p=0.0001)$ and $\mathrm{B}-1 v s . \mathrm{B}+3(p=0.0209) .50 \%$ of the patients with $R>0.4(R=\mathrm{CTC}$ ratio of $\mathrm{B}+3$ over $\mathrm{B} 0)$ showed tumor recurrence within 1 year after surgery, whereas the probability was only $14.3 \%$ for patients with $R$ $<0.4(p=0.043)$. The prognostic utility of CTCs in $\mathrm{EC}$ has been shown also in studies where the gene expression of survivin, ERCC1, and APC has been tested by RT-PCR, as shown in Table 2 .

The prognostic relevance of the presence of DTCs in bone marrow (BM) for the postoperative course of EC has also been evaluated recently [18]: 370 patients with EC diagnosis (189 squamous cell carcinomas and 181 adenocarcinomas), were surgically treated with complete resection (R0). They received neither adjuvant nor neoadjuvant therapy. DTCs were detected by an immunocytochemical cytokeratin assay in preoperatively taken BM aspirates. Overall, 120 (32.4\%) patients harbored DTCs in their BM. The presence of DTCs significantly correlated with aggressive tumor biology, as indicated by increased tumor size $(p=0.026)$, regional $(p=0.002)$ and distant $(p=0.012)$ lymph node metastases, and higher relapse rate $\left(p<0.001, \chi^{2}\right.$ test). The presence of DTCs in bone marrow was a very strong and independent prognostic factor in patients with resectable EC [18].

The CTC status in the PB of patients with esophageal squamous cell carcinoma (ESCC), before and after radiotherapy (RT), was evaluated by Yin et al. [19]. A total of 72 ESCC patients enrolled in this study were treated with radical RT. The nested RT-PCR reaction was used to detect the three representative markers of CTCs: CEA, CK19, and survivin. The results showed that the presence of CTCs, and the positive expression of at least one of these three markers in patients with ESCC pre-RT and post-RT were $54.2 \%$ and $38.9 \%$, respectively $(p=0.059)$. Furthermore, the analysis of the patients according to lymph node metastasis and adverse 2-year progression -free survival (PFS) revealed changes in CTC status after RT, which would reflect patients' response to RT. In a multivariate analysis with the Cox proportional hazard model, only CTC positivity post-RT was an independent, unfavorable prognostic factor for ESCC, apart from subsequent chemotherapy and patients' Karnofsky performance status scores (a scale quantifying cancer patients' general well-being). In conclusion, the positive detection of CTCs in patients with ESCC after RT may be a promising biomarker for radiation efficiency and prognosis assessment in ESCC [19].

\section{Gastric cancer (GC)}

Follow-up studies on gastric cancer (GC) patients suggested that CTC-positive cases with increased burdens of CTCs were associated with poorer prognoses than CTC-negative cases. The situation was similar with DTCs [15]. Both localized and metastatic GC can shed detectable concentrations of CTCs into the blood. The presence of CTCs in circulation suggests not only a high risk of tumor recurrence, but also an unfavorable clinical outcome even in the early stages of GC [20]. The prognostic impact of CTCs in GC has been reported in several studies [21-27]. The sensitivity of RT-PCR CTC detection was superior to the other less commonly used cytological detection methods involving fluorescence-activated cell sorting (FACS), immunohistochemistry (IHC), and immunocytochemistry (ICC) [20]. For the identification of CTCs in GC, different markers and their combinations were tested in the analyzed studies. The combination of EpCAM, CK8, CK18, and CK19 seems to be prognostically the most relevant in GC [7, 24]. On the other side, single survivin expression also achieved prognostic significance in at least 2 studies [29, 30]. Based on the analyzed data, detection of CTCs might be used as a noninvasive method, not only for the confirmation of GC diagnosis, but also for estimation of prognosis.

\section{Colorectal cancer (CRC)}

In general, the detection of CTCs in colorectal cancer (CRC), independently of the method and markers 
used, correlates with the stage of the cancer disease $[31,32]$. On the other side, the correlation of CTCs with some known clinicopathological prognostic factors (e.g., T4 tumor size, perineural invasion, bowel obstruction, high preoperative CEA levels) is still uncertain [32]. It is believed that the correlation of CTCs with clinicopathological factors would increase if the sensitivity of the CTC detection were higher. CTC positivity is observed in approximately 40-50\% of metastatic CRC patients. Differences in CTCs detection can be observed depending on the sampling site, as shown by Rahbari et al. [10], who tested compartmental differences of CTC in CRC. The qualitative and quantitative detection of CTCs was higher in the mesenteric venous blood (MVB) than in the central venous blood (CVB) of patients with CRC. It has been speculated that the liver works as a filter and stops CTCs from entering the central circulation [6]. Moreover, higher counts of CTCs were detected when the tumor was localized in the lower part of rectum than in the cases of middle and high rectal involvement $[6,33]$.

The biomarkers used for the CTCs detection in cytological or RT-PCR examination of patients with CRC are listed in Tables 3 and 4. Generally, the EpCAM pre-enrichment is a basis for further cytokeratine (CK19/20, CK8/18) and CEA testing. Recently, plastin 3 has been shown to have significant clinical relevance. Plastin3 positivity in the PB was found to be associated with clinicopathological risk factors, such as depth of invasion, lymph node and liver metastasis, presence of peritoneal dissemination, increased recurrence rate, and higher Dukes stage. It is very important to note that plastin 3 expression was also detected in all patients with recurrent disease, and at a level higher than in the case of prerecurrence and of patients without recurrence [34]. The correlation between CTCs and prognosis in CRCs was stronger if CKs and multiple markers were used than for the one-marker assay [35].

Recently, several meta-analyses evaluating the prognostic value of CTC examination in CRC have been published. Rahbari et al. [36] included 36 studies and 3094 patients in their final meta-analysis. The pooled analyses combining all sampling sites (PB, mesenteric PB (MPB), and BM) associated the detection of CTCs/DTCs with poor recurrence-free survival (RFS). Stratification by sampling site showed that detection of CTCs in the PB compartment was a statistically significant prognostic factor, but that detection in the MPB or BM was not.

Similarly, 12 studies representing 1329 patients were suitable for pooled analysis of CRC patients in a prognostic study [37]. The OS and PFS were worse in CTC-positive patients, whereas analyzing PFS separately, the subgroup with significantly worse survival rate contained over 35\% CTC-positive patients. Multivariate analysis was performed on eight studies and identified the detection of CTCs as an independent prognostic factor for survival. Moreover, the meta-analysis reported that the detection of CTCs in PB of patients with resectable colorectal liver metastases, or with widespread metastatic CRC, was associated with disease progression and poor survival [37]. The study of Katsuno et al. [38] highlights the potential importance of cancer cell detection in the venous drainage of colorectal cancers as a prognostic marker and a mode of staging in this neoplastic disease.

Regarding the effect of chemotherapy on CTC counts, it has been evaluated that the prognosis of patients with undetectable CTCs after chemotherapy was significantly better [39]. Additionally, molecular detection of persistent postoperative CTCs has been confirmed as a prognostic marker of early relapse in I-III stage CRC patients, which could help to select patients for an enhanced follow-up and therapeutic program [40, 41].

In summary, it is expected that CTCs and DTCs will be used for mutational analysis of the genes connected directly to the targeted therapy (e.g., $K R A S$, $B R A F)$. The heterogeneity of the genetic profiles of cells from the primary tumor, metastatic tumors, and CTCs may be an explanation for the variable response to EGFR-inhibitor chemotherapy [5, 42, 43]. CTCs are not only a marker for advanced disease, but also have prognostic and predictive potential. A decrease in CTC levels during chemotherapy is correlated with improved responses to chemotherapy [39].

Several very important questions need to be answered, and further studies are required to unify the isolation techniques before CTCs can be adapted for widespread clinical use. In particular, the following questions should be central to future research: Can CTCs be used to define a group of patients with "resectable" metastases who should not undergo resection? Can CTCs be used to monitor the immediate effectiveness of systemic chemotherapy or to predict which chemotherapy would be most effective? Can CTCs be used to help staging patients with metastatic CRCs?

Finally, we are reminded that stage IV of colorectal cancer is a disease with many possible outcomes, ranging from rapid death to recovery [40]. We also recall that our ability to predict which patient will experience which outcome is relatively limited. The detection of CTCs is a potentially promising biomarker that could contribute to the staging of the cancer and this deserves a prospective study. 


\section{Conclusion}

In summary, it is essential to establish sensitive, specific technologies to detect CTCs. More detailed analyses of their molecular characteristics should be performed with the aim of understanding the biology of CTCs and DTCs. This may provide a yet-untapped option to develop therapeutic strategies that will effectively treat and prevent metastatic process for each person individually.

\section{Acknowledgements}

This study was supported by a grant (entitled "Sledování hladiny CTC u vybraných nádorů gastrointestinálního traktu”) provided by the League Against Cancer, Prague, Czech Republic Grant, and by a grant from the Czech Ministry of Health (IGA NT14439-3/2013).

\section{References}

1. Steinert G, Schölch S, Koch M et al. Biology and significance of circulating and disseminated tumor cells in colorectal cancer. Langenbecks Arch Surg. 2012;397:535-542.

2. Brechot $\mathrm{C}$ et al. Molecular analysis of circulating tumor cells isolated by ISET: A diagnostic approach to improve follow up and treatment of patients with cancer. Boston, World CTC Summit, 2012.

3. Takeuchi H, Kitagawa Y. Detection of circulating tumor cells in gastrointestinal cancer: Has its time come? J Gastrointest Oncol. 2012;3:84-85.

4. Maheswaran S, Haber DA. Circulating tumor cells: a window into cancer biology and metastasis. Curr Opin Genet Dev. 2010;20:96-99.

5. Kin C, Kidess E, Poultsides GA et al. Colorectal cancer diagnostics: biomarkers, cell-free DNA, circulating tumor cells and defining heterogeneous populations by single-cell analysis. Expert Rev Mol Diagn. 2013;13:581-599.

6. Negin BP, Cohen SJ. Circulating tumor cells in colorectal cancer: past, present, and future challenges. Curr Treat Options Oncol. 2010;11:1-13.

7. Hiraiwa K, Takeuchi H, Hasegawa H et al. Clinical significance of circulating tumor cells in blood from patients with gastrointestinal cancers. Ann Surg Oncol. 2008;15:39092-39100.

8. Moreno JG, O'Hara SM, Gross S et al. Changes in circulating carcinoma cells in patients with metastatic prostate cancer correlate with disease status. Urology. 2001;58:386-392.

9. Dong X, Alpaugh KR, Cristofanilli M. Circulating tumor cells (CTCs) in breast cancer: a diagnostic tool for prognosis and molecular analysis. Chin J Cancer Res. 2012;24:388-398.

10. Rahbari NN, Bork U, Kircher A et al. Compartmental Differences of Circulating Tumor Cells in Colorectal Cancer. Ann Surg Oncol. 2012;19:2195-2202.

11. Iwanicki-Caron I, Basile P, Toure E et al. Usefulness of Circulating Tumor Cell Detection in Pancreatic Adenocarcinoma Diagnosis. Am J Gastroenterol. 2013;108:152-155.

12. Juratli MA, Sarimollaoglu M, Siegel E et al. Real-time monitoring of circulating tumor-cell release during tumor manipulation using in vivo photoacoustic and fluorescent flow cytometry. Head Neck. 2013; DOI: 10.1002/hed.23439. [Epub ahead of print].
13. Butler TP, Gullino PM. Quantitation of cell shedding into efferent blood of mammary adenocarcinoma. Cancer Res. 1975;35:512-516.

14. Jiao LR, Apostolopoulos C, Jacob J et al. Unique localization of circulating tumor cells in patient with hepatic metastases. J Clin Oncol. 2009;36:6160-6165.

15. Wang GY, Li Y, Yu YM et al. Detection of disseminated tumor cells in bone marrow of gastric cancer using magnetic activated cell sorting and fluorescent activated cell sorting. $J$ Gastroenterol Hepatol. 2009;24:299-306.

16. Katayama A, Mafune K, Tanaka Y et al. Autopsy findings in patients after curative esophagectomy for esophageal carcinoma. J Am Coll Surg. 2003;196:866-873.

17. Liu Z, Jiang M, Zhao J et al. Circulating tumor cells in perioperative esophageal cancer patients: quantitative assay system and potential clinical utility. Clin Cancer Res. 2007;13:2992-2997.

18. Vashist YK, Effenberger KE, Vettorazzi E et al. Disseminated tumor cells in bone marow and the natural course of resected esophageal cancer. Ann Surg. 2012;255:1105-1112.

19. Yin XD, Yuan X, Xue JJ et al. Clinical significance of carcinoembryonic antigen-, cytokeratin 19-, or survivin-positive circulating tumor cells in the peripheral blood of esophageal squamous cell carcinoma patients treated with radiotherapy. Dis Esophagus. 2012;25:750-756.

20. Zhang ZY, Ge HY. Micrometastasis in gastric cancer. Cancer Lett. 2013;336:34-45.

21. Arigami T, Uenosono $\mathrm{Y}$, Hirata $\mathrm{M}$ et al. B7-H3 expression in gastric cancer: a novel molecular blood marker for detecting circulating tumor cells. Cancer Sci. 2011;102:1019-1024.

22. Saad AA, Awed NM, Abd Elkerim NN et al. Prognostic significance of E-cadherin expression and peripheral blood micrometastasis in gastric carcinoma patients. Ann Surg Oncol. 2010;17:3059-3067.

23. Pituch-Noworolska A, Kolodziejczyk P, Kulig J et al. Circulating tumour cells and survival of patients with gastric cancer. Anticancer Res. 2007;27:635-640.

24. Yeh KH, Chen YC, Yeh SH et al. Detection of circulating cancer cells by nested reverse transcription-polymerase chain reaction of cytokeratin-19 (K19)-possible clinical significance in advanced gastric cancer. Anticancer Res. 1998;18:1283-1286.

25. Koga T, Tokunaga E, Sumiyoshi Y et al. Detection of circulating gastric cancer cells in peripheral blood using real time quantitative RT-PCR. Hepatogastroenterology. 2008;84:1131-1135.

26. Illert B, Fein M, Otto $\mathrm{C}$ et al. Disseminated tumor cells in the blood of patients with gastric cancer are an independent predictive marker of poor prognosis. Scand J Gastroenterol. 2005;40:843-849.

27. Uen $\mathrm{YH}, \mathrm{Lin} \mathrm{SR}, \mathrm{Wu} \mathrm{CH}$ et al. Clinical significance of MUC1 and c-Met RT-PCR detection of circulating tumor cells in patients with gastric carcinoma. Clin Chim Acta. 2006;367:55-61.

28. Matsusaka S, Chìn K, Ogura $\mathrm{M}$ et al. Circulating tumor cells as a surrogate marker for determining response to chemotherapy in patients with advanced gastric cancer. Cancer Sci. 2010;101:1067-1071.

29. Bertazza L, Mocellin S, Marchet A et al. Survivin gene levels in the peripheral blood of patients with gastric cancer independently predict survival. $J$ Transl Med. 2009;7:111.

30. Cao W, Yang W, Li H et al. Using detection of survivin-expressing circulating tumor cells in peripheral blood to predict tumor recurrence following curative resection of gastric cancer. J Surg Oncol. 2011;103:110-115.

31. Cohen SJ, Alpaugh RK, Gross S et al. Isolation and characterization of circulating tumor cells in patients with metastatic colorectal cancer. Clin Colorectal Cancer. 2006;6:125-132. 
32. Sastre J, Maestro ML, Gómez-Espa a A et al. Circulating tumor cell count is a prognostic factor in metastatic colorectal cancer patients receiving first-line chemotherapy plus bevacizumab: a Spanish Cooperative Group for the Treatment of Digestive Tumors study. Oncologist. 2012;17:947-955

33. Molnar B, Ladanyi A, Tanko L et al. Circulating tumor cell clusters in the peripheral blood of colorectal cancer patients. Clin Cancer Res. 2001;12:4080-4085.

34. Yokobori T, Iinuma $\mathrm{H}$, Shimamura $\mathrm{T}$ et al. Plastin 3 is a novel marker for circulating tumor cells undergoing the epithelial-mesenchymal transition and is associated with colorectal cancer prognosis. Cancer Res. 2013;73:2059-2069.

35. Torino F, Bonmassar E, Bonmassar L et al. Circulating tumor cells in colorectal cancer patients. Cancer Treat Rev. 2013;39:759-772.

36. Rahbari NN, Aigner M, Thorlund K et al. Meta-analysis shows that detection of circulating tumor cells indicates poor prognosis in patients with colorectal cancer. Gastroenterology. 2010;138:1714-1726.

37. Groot Koerkamp B, Rahbari NN, Büchler MW et al. Circulating tumor cells and prognosis of patients with resectable colorectal liver metastase or widespread metastatic colorectal cancer: a meta-analysis. Ann Surg Oncol. 2013;20:2156-2165.

38. Katsuno H, Zacharakis E, Aziz O et al. Does the presence of circulating tumor cells in the venous drainage of curative colorectal cancer resections determine prognosis? A meta-analysis. Ann Surg Oncol. 2008;15:3083-3091.

39. Neki K, Kawahara H, Watanabe K et al. Usefulness of circulating tumor cells after preliminary chemotherapy for prediction of response to further anticancer therapy in patients with initially unresectable metastatic colorectal cancer. Anticancer Res. 2013;33:1769-1772.

40. Uen YH, Lu CY, Tsai HL et al. Persistent presence of postoperative circulating tumor cells is a poor prognostic factor for patients with stage I-III colorectal cancer after curative resection. Ann Surg Oncol. 2008;15:2120-2128.

41. Lu CY, Uen YH, Tsai HL et al. Molecular detection of persistent postoperative circulating tumour cells in stages II and III colon cancer patients via multiple blood sampling: prognostic significance of detection for early relapse. $\mathrm{Br}$ J Cancer. 2011;104:1178-1184.

42. Lankiewicz S, Zimmermann S, Hollmann C et al. Circulating tumour cells as a predictive factor for response to systemic chemotherapy in patients with advanced colorectal cancer. Mol Oncol. 2008;3:349-355.

43. Gazzaniga P, Gradilone A, Petracca A et al. Molecular markers in circulating tumour cells from metastatic colorectal cancer patients. J Cell Mol Med. 2010;14:2073-2077.

44. Kolodziejczyk P, Pituch-Noworolska A, Drabik G et al. The effects of preoperative chemotherapy on isolated tumor cells in the blood and bone marrow of gastric cancer patients. $\mathrm{Br}$ J Cancer. 2007;97:589-592.

45. de Albuquerque A, Kubisch I, Ernst D et al. Development of a molecular multimarker assay for the analysis of circulating tumor cells in adenocarcinoma patients. Clin Lab. 2012;58:373-384.

46. Hoffmann AC, Vallböhmer D, Prenzel K et al. Methylated DAPK and APC promoter DNA detection in peripheral blood is significantly associated with apparent residual tumor and outcome. J Cancer Res Clin Oncol. 2009;135:1231-1237.

47. Brabender J, Vallböhmer D, Grimminger P et al. ERCC1 RNA expression in peripheral blood predicts minor histopathological response to neoadjuvant radio-chemotherapy in patients with locally advanced cancer of the esophagus. J Gastrointest Surg. 2008;12:1815-1821.
48. Hoffmann AC, Warnecke-Eberz U, Luebke T et al. Survivin mRNA in peripheral blood is frequently detected and significantly decreased following resection of gastrointestinal cancers. J Surg Oncol. 2007;95:51-54.

49. Ikoma D, Ichikawa D, Ueda $\mathrm{Y}$ et al. Circulating tumor cells and aberrant methylation as tumor markers in patients with esophageal cancer. Anticancer Res. 2007;27:535-539.

50. Hoffmann AC, Warnecke-Eberz U, Luebke T et al. Survivin mRNA in peripheral blood is frequently detected and significantly decreased following resection of gastrointestinal cancers. J Surg Oncol. 2007;95:51-54.

51. Ikeguchi M, Kaibara N. Detection of circulating cancer cells after a gastrectomy for gastric cancer. Surg Today. 2005;35:436-441.

52. Ito H, Kanda T, Nishimaki T et al. Detection and quantification of circulating tumor cells in patients with esophageal cancer by real-time polymerase chain reaction. J Exp Clin Cancer Res. 2004;23:455-464.

53. Kaganoi J, Shimada Y, Kano M et al. Detection of circulating oesophageal squamous cancer cells in peripheral blood and its impact on prognosis. Br J Surg. 2004;91:1055-1060.

54. Huang P, Wang J, Guo Y et al. Molecular detection of disseminated tumor cells in the peripheral blood in patients with gastrointestinal cancer. J Cancer Res Clin Oncol. 2003;129:192-198.

55. Nakashima S, Natsugoe S, Matsumoto $M$ et al. Clinical significance of circulating tumor cells in blood by molecular detection and tumor markers in esophageal cancer. Surgery. 2003;133:162-169.

56. Koike M, Hibi K, Kasai Y et al. Molecular detection of circulating esophageal squamous cell cancer cells in the peripheral blood. Clin Cancer Res. 2002;8:2879-2882.

57. Miyazono F, Natsugoe S, Takao S et al. Surgical maneuvers enhance molecular detection of circulating tumor cells during gastric cancer surgery. Ann Surg. 2001;233:189-194.

58. Soeth E, Vogel I, Röder C et al. Comparative analysis of bone marrow and venous blood isolated from gastrointestinal cancer patients for the detection of disseminated tumor cells using reverse transcription PCR. Cancer Res. 1997;57:3106-3110.

59. Galletti G, Sung MS, Vahdat LT et al. Isolation of breast cancer and gastric cancer circulating tumor cells by use of an anti HER2-based microfluidic device. Lab Chip. 2014;14:147-156.

60. Sato N, Hayashi N, Imamura Y et al. Usefulness of Transcription-Reverse Transcription Concerted Reaction Method for Detecting Circulating Tumor Cells in Patients With Colorectal Cancer. Ann Surg Oncol. 2012;19:2060-2065.

61. Aggarwal C, Meropol NJ, Punt CJ et al. Relationship among circulating tumor cells, CEA and overall survival in patients with metastatic colorectal cancer. Ann Oncol. 2013;24:420-428.

62. Flatmark K, Borgen E, Nesland JM et al. Disseminated tumour cells as a prognostic biomarker in colorectal cancer. Br J Cancer. 2011;104:1434-1439.

63. Matsusaka S, Suenaga M, Mishima Y et al. Circulating endothelial cells predict for response to bevacizumab-based chemotherapy in metastatic colorectal cancer. Cancer Chemother Pharmacol. 2011;68:763-768.

64. Tol J, Koopman M, Miller MC et al. Circulating tumour cells early predict progression-free and overall survival in advanced colorectal cancer patients treated with chemotherapy and targeted agents. Ann Oncol. 2010;5:1006-1012.

65. Papavasiliou P, Fisher T, Kuhn J et al. Circulating tumor cells in patients undergoing surgery for hepatic metastases from colorectal cancer. Baylor Univ Med Cent Proc. 2010;23:11-14. 
66. Königsberg R, Gneist M, Jahn-Kuch D et al. Circulating tumor cells in metastatic colorectal cancer: efficacy and feasibility of different enrichment methods. Cancer Lett. 2010;293:117-123.

67. Marrinucci D, Bethel K, Lazar D et al. Cytomorphology of Circulating Colorectal Tumor Cells: A Small Case Series. J Oncol. 2010;2010:861341.

68. Maestro LM, Sastre J, Rafael SB et al. Circulating Tumor Cells in Solid Tumor in Metastatic and Localized Stages. Anticancer Res. 2009;29:4839-4843.

69. Wong SC, Chan CM, Ma BB et al. Clinical significance of cytokeratin 20-positive circulating tumor cells detected by a refined immunomagnetic enrichment assay in colorectal cancer patients. Clin Cancer Res. 2009;15:1005-1012.

70. Cohen SJ, Punt CJ, Iannotti N et al. Relationship of Circulating Tumor Cells to Tumor Response, Progression-Free Survival, and Overall Survival in Patients With Metastatic Colorectal Cancer. J Clin Oncol. 2008;26:3213-3221.

71. Schoppmeyer K, Frühauf N, Oldhafer K et al. Tumor cell dissemination in colon cancer does not predict extrahepatic recurrence in patients undergoing surgery for hepatic metastases. Oncol Rep. 2006;15:449-454.

72. Barbazán J, Alonso-Alconada L, Muinelo-Romay L et al. Molecular Characterization of Circulating Tumor Cells in $\mathrm{Hu}-$ man Metastatic Colorectal Cancer. PLoS One. 2012;7:e40476.

73. Iinuma $\mathrm{H}$, Watanabe $\mathrm{T}$, Mimori $\mathrm{K}$ et al. Clinical significance of circulating tumor cells, including cancer stem-like cells, in peripheral blood for recurrence and prognosis in patients with Dukes' stage B and C colorectal cancer. J Clin Oncol. 2011;29:1547-1555.

74. Vogelaar FJ, Mesker WE, Rijken AM et al. Clinical impact of different detection methods for disseminated tumor cells in bone marrow of patients undergoing surgical resection of colorectal liver metastases: a prospective follow-up study. BMC Cancer. 2010;10:153.

75. Yen LC, Yeh YS, Chen CW et al. Detection of KRAS oncogene in peripheral blood as a predictor of the response to cetuximab plus chemotherapy in patients with metastatic colorectal cancer. Clin Cancer Res. 2009;15:4508-4513.

76. Koyanagi K, Bilchik AJ, Saha S et al. Prognostic Relevance of occulat nodal micrometastases and circulating tumor cells in colorectal cancer in a prospective multicenter trial. Cli Cancer Res. 2008;22:7391-7396.
77. Zieglschmid V, Hollmann C, Mannel J et al. Tumor-associated gene expression in disseminated tumor cells correlates with disease progression and tumor stage in colorectal cancer. Anticancer Res. 2007;27:1823-1832.

78. Allen-Mersh TG, McCullough TK, Patel $\mathrm{H}$ et al. Role of circulating tumour cells in predicting recurrence after excision of primary colorectal carcinoma. Br J Surg. 2007;94:96-105.

79. Wang JY, Lin SR, Wu DC et al. Multiple molecular markers as predictors of colorectal cancer in patients with normal perioperative serum carcinoembryonic antigen levels. Clin Canc Res. 2007;13:2406-2413.

80. Sadahiro S, Suzuki T, Maeda Y et al. Detection of carcinoembryonic antigen messenger RNA-expressing cells in peripheral blood 7 days after curative surgery is a novel prognostic factor in colorectal cancer. Ann Surg Oncol. 2007;14:1092-1098.

81. Iinuma $\mathrm{H}$, Okinaga $\mathrm{K}$, Egami $\mathrm{H}$ et al. Usefulness and clinical significance of quantitative real-time RT-PCR to detect isolated tumor cells in the peripheral blood and tumor drainage blood of patients with colorectal cancer. Int J Oncol. 2006;28:297-306.

82. Koch M, Kienle P, Hinz U et al. Detection of hematogenous tumor cell dissemination predicts tumor relapse in patients undergoing surgical resection of colorectal liver metastases. Ann Surg. 2005;241:199-205.

83. Staritz P, Kienle P, Koch M et al. Detection of disseminated tumour cells as a potential surrogate-marker for monitoring palliative chemotherapy in colorectal cancer patients. J Exp Clin Cancer Res. 2004;23:633-639.

84. Vlems FA, Diepstra JH, Punt CJ et al. Detection of disseminated tumour cells in blood and bone marrow samples of patients undergoing hepatic resection for metastasis of colorectal cancer. Br J Surg. 2003;90:989-995.

85. Ito $\mathrm{S}$, Nakanishi $\mathrm{H}$, Hirai $\mathrm{T}$ et al. Quantitative detection of CEA expressing free tumor cells in the peripheral blood of colorectal cancer patients during surgery with real-time RT-PCR on a LightCycler. Cancer Lett. 2002;183:195-203.

86. Patel H, Le Marer N, Wharton RQ et al. Clearance of circulating tumor cells after excision of primary colorectal cancer. Ann Surg. 2002;235:226-231.

87. Wyld DK, Selby P, Perren TJ et al. Detection of colorectal cancer cells in peripheral blood by reverse-transcriptase polymerase chain reaction for cytokeratin 20. Int J Cancer. 1998;79:288-293.

Submitted: 22 December, 2013

Accepted after reviews: 29 December, 2013 